\title{
Measurements of differential cross-sections for top-quark pair production in association with additional jets in highly boosted events using the ATLAS detector
}

\author{
Jonathan Jamieson ${ }^{a, *}$ on behalf of the ATLAS Collaboration \\ ${ }^{a}$ University of Glasgow, \\ University Avenue, Glasgow, United Kingdom \\ E-mail: jonathan.jamieson@cern.ch
}

\begin{abstract}
Top anti-top pair production at the Large Hadron Collider is often observed in the presence of additional high energy radiation. Similarly abundant top-quark pair production allows us to probe high transverse momentum top quarks. These proceedings showcase recent top-quark pair production cross-section measurements with a high energy (boosted) top-quark using proton-proton collision events at a centre-of-mass energy of $13 \mathrm{TeV}$ recorded by the ATLAS detector from 2015 to 2018. The results are compared to different QCD predictions showcasing how suited current theories are to estimating complex, multi-scale, and above leading order processes. Differential top-quark pair cross-section measurements as functions of various event kinematic properties are presented, using events with a high transverse momentum large-R jet, one muon or electron and at least two $b$-tagged jets in the final-state. The sensitivity of these measurements to beyond Standard Model physics effects is also probed by imposing simultaneous limits on two effective field theory operators.
\end{abstract}

\footnotetext{
*** The European Physical Society Conference on High Energy Physics (EPS-HEP2021), ***

*** 26-30 July $2021 * * *$

*** Online conference, jointly organized by Universität Hamburg and the research center DESY ***
}

\footnotetext{
${ }^{*}$ Speaker
} 


\section{Introduction}

The high rate of top anti-top $(t \bar{t})$ production at the Large Hadron Collider (LHC) and the sensitivity of the top-quark sector to beyond Standard Model (SM) effects motivate making precise differential $t \bar{t}$ cross-section measurements of high transverse momentum $\left(p_{\mathrm{T}}\right)$ top-quarks. These proceedings summarise results from the following differential cross-section measurement [1], in which $t \bar{t}$ events containing a high $p_{\mathrm{T}}$, hadronically-decaying top quark are selected and differential cross-sections as a function of various kinematic distributions are measured. A novel approach is used to reduce the impact of jet uncertainties, and the results are compared to available SM predictions at next-to-leading order accuracy (NLO) and the same predictions re-weighted to match calculations of $t \bar{t}$ production at next-to-next-to-leading order accuracy (NNLO) [2]. The measured top-quark $p_{\mathrm{T}}$ is used to test sensitivity to physics beyond the SM using a model-independent effective field theory (EFT) framework. Simultaneous limits are set on two effective operators, $O_{t G}$ and $O_{t q}^{(8)}$.

\section{Event selection}

Data events were recorded by the ATLAS detector [3] in $p p$ collisions at $\sqrt{s}=13 \mathrm{TeV}$ from 2015-18, corresponding to an integrated luminosity of $139 \mathrm{fb}^{-1}$. Various Monte Carlo (MC) simulated event samples, summarised in [1], are used to determine background contributions, derive corrections for detector effects, simulate potential new physics contributions, and to provide comparisons with data. Final state products of the $t \bar{t}$ decay; electrons, muons, hadronic jets and missing energy $\left(E_{\mathrm{T}}^{\text {miss }}\right)$, are reconstructed from signals within the ATLAS detector in the standard way. Highly boosted top-quarks ( $p_{\mathrm{T}} \gtrsim 2 m_{\text {top }}$ ) that decay hadronically are reconstructed as large radius re-clustered (RC) jets using the anti- $k_{t}$ [4] clustering algorithm with radius parameter $R=1.0$ and the collection of $R=0.4$ jets as input. Events are selected such that they contain exactly one electron or exactly one muon, at least two $b$-tagged jets and one top-tagged RC jet. The top-tagged jet corresponds to the highest $p_{\mathrm{T}} \mathrm{RC}$ jet in the event which contains at least one $b$-jet and has $p_{\mathrm{T}}>355 \mathrm{GeV}$ and $120<$ mass $<220 \mathrm{GeV}$. Additional selection criteria are applied to improve the signal purity and the selected objects are then used to reconstruct the kinematics of the $t \bar{t}$ system. Similar selections are applied at particle-level to define a fiducial region for the measurement.

\section{Cross-section measurement}

The strategy used to measure differential cross-sections is to first correct the small radius jet energies in data by a jet energy scale factor (JSF) as discussed in Section 3.1. The number of events after that correction $\left(N_{\mathrm{d}}(\mathrm{JSF})\right)$ then have the background contributions $\left(N_{\mathrm{b}}\right)$ subtracted. The main backgrounds are expected to be from $t W$ single top-quark production and $W+$ jets production. The background-subtracted distributions are then corrected (unfolded) for detector effects in three stages, first $t \bar{t}$ events that do not pass the fiducial requirements are corrected for with the factor $f_{\text {acc }}$. An iterative Bayesian unfolding [5] (denoted with $\mathcal{M}^{-1}$ ) is then used to correct for the limited resolution of the detector. Lastly the factor $f_{\text {eff }}$ corrects for events that pass the fiducial requirements but do not pass the detector-level event selection. The unfolded distributions are further corrected for the integrated luminosity $(\mathcal{L})$ and bin width $(\Delta X)$ giving the differential cross-section at particle-level:

$$
\frac{d \sigma}{d X}=\frac{1}{\mathcal{L} \cdot \Delta X} \frac{1}{f_{\mathrm{eff}}} \mathcal{M}^{-1} f_{\mathrm{acc}}\left(N_{d}(\mathrm{JSF})-N_{b}\right) .
$$




\subsection{Correction of the jet energy}

The data and simulation are assumed to have an overall difference in jet energy scale that is parameterised with a jet energy scale factor (JSF). The analysis includes a measurement of JSF using the reconstructed invariant mass of the selected hadronically decaying top-quark ( $m^{\text {top }}$ had) in conjunction with the known value of the top quark mass. This process reduces the impact of uncertainties related to the jet energy scale. Figure 1a shows the $m^{\text {top had }}$ distribution for three different JSF values obtained by applying the JSF to the energy of every jet in the nominal $t \bar{t}$ sample and simulated background estimates. Figure $1 \mathrm{~b}$ shows that the mean of the $m^{\text {top }}$ had distribution is linearly dependent on JSF. The mean value in data is used to determine the JSF from this linear relationship giving a value of $1.00035 \pm 0.00087$ [1], where the uncertainty is due to the number of observed data events. The JSF correction is then applied to the data and the event selection is reapplied, yielding the corrected distributions that are used as input for the cross-section calculation.

\subsection{Systematic uncertainties}

Systematic uncertainties affect the measured cross-section through the unfolding corrections. Each systematic is evaluated by creating pseudo-data where the source of uncertainty has been varied and then the full analysis procedure, including extracting a JSF value, is repeated. The impact of each uncertainty is assessed by comparing the unfolded pseudo-data to the relevant particle-level prediction. The modelling uncertainties generally have the largest impact on the measurement, while the jet and $b$-tagging uncertainties are important in particular phase space regions. The impact of the JSF correction procedure is evaluated by comparing the impact of each source for the case where no JSF correction is applied with the nominal analysis setup. A significant improvement in the precision is seen due to the JSF correction, as shown in Figure 1c, which displays the effect of the JSF correction on the uncertainty for the invariant mass of the two top-quarks, both for the total uncertainty and the jet energy scale (JES) uncertainty.

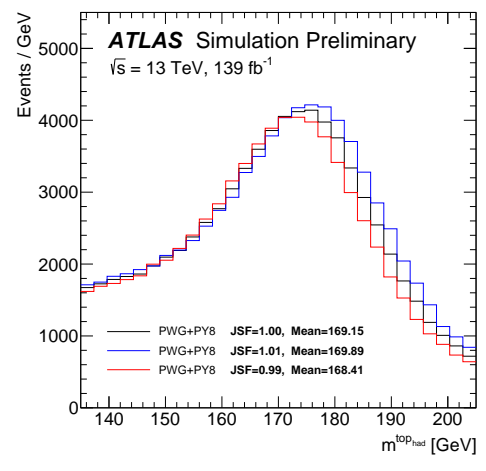

(a)

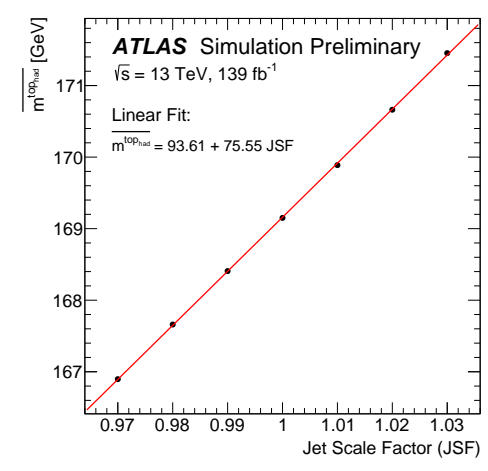

(b)

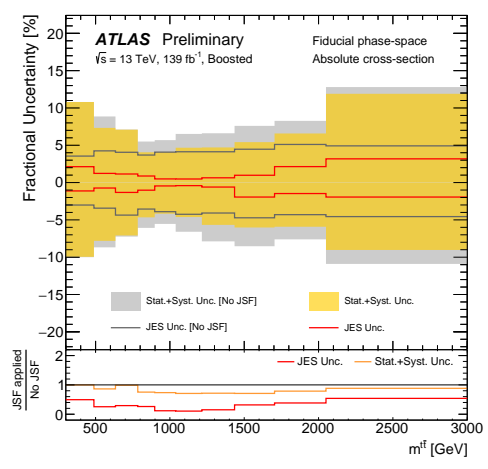

(c)

Figure 1: (a) The distribution of the invariant mass of the top-tagged jet $\left(m^{\text {top }}\right.$ had $)$ for the expected signal plus background for three example values of the JSF. (b) The mean of the same distribution as a function of JSF and a linear fit to the simulated samples. (c) Effect of the JSF correction on the cross-section uncertainty as a function of $m^{t \bar{t}}$. The coloured (grey) bands and lines represents the labelled uncertainty with (without) the JSF correction applied. The bottom panel shows the ratios of the absolute size of the uncertainties with and without the JSF correction applied. [1] 


\section{Results}

The inclusive fiducial cross-section is measured to be $1.267 \pm 0.005$ (stat) $\pm 0.053 \mathrm{pb}$ (syst) [1] . The relative precision of $4.2 \%$ is smaller than the uncertainty on the theoretical calculation at NNLO+NNLL of $6.1 \%\left(4.2 \% \mathrm{PDF} \oplus 3 \%\right.$ scale $\left.\oplus 2.8 \% \mathrm{~m}_{\mathrm{t}}\right)$ [6], as shown in Figure 2a. All MC setups give higher predictions than the data however the agreement is seen to improve significantly after re-weighting to the NNLO prediction. The same improvement is observed for the differential distributions however no single generator setup is able to accurately describe all measured variables even with the re-weighting. In particular, details of the additional radiation are not well described by any of the MC predictions as shown in Figure $2 \mathrm{~b}$ which compares the measured and simulated cross-section results for the leading additional jet $p_{\mathrm{T}}$. The shape of the distribution differs noticeably between the MADGRAPH5_aMC@NLO and Powheg setups, indicating that the technique for matching the matrix element and the parton shower is a relevant issue. The precision of the results in general indicates that comparisons with higher precision generators would be beneficial.

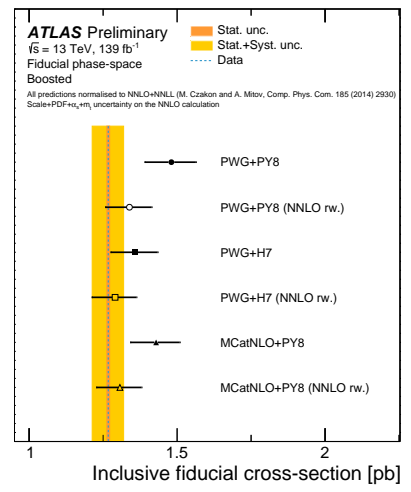

(a)

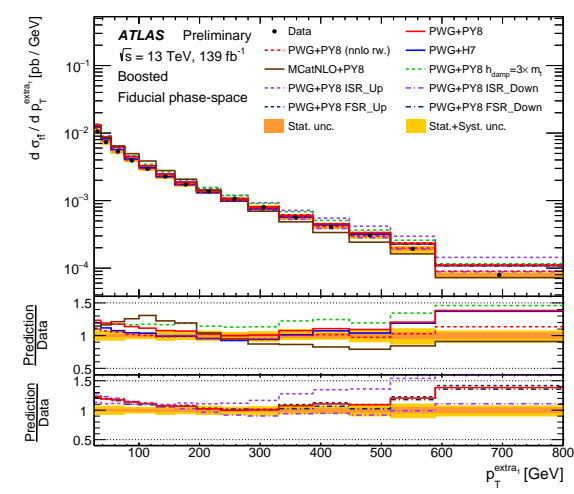

(b)

Figure 2: (a) The fiducial cross-section at particle-level for boosted $t \bar{t}$ production measured in data (dashed line) is compared to several NLO predictions with (open markers) and without (closed markers) the NNLO re-weighting applied. (b) Differential cross-section measurement for the leading additional jet $p_{\mathrm{T}}$, comparing data to various MC simulations. The lower panels display the ratios between prediction and data. In both plots the yellow (orange) band represents the total (statistical) uncertainty on the measured cross-section. [1]

\section{Limits on EFT operators}

The sensitivity of the analysis to potential new physics is explored by interpreting the $p_{\mathrm{T}}^{\text {top }}$ had distribution in terms of dimension six operators within the EFT framework [7] with new physics scale $\Lambda=1 \mathrm{TeV}$. The operators, parameterised by Wilson coefficients $C_{t G}$ and $C_{t q}^{(8)}$, affect different aspects of $t \bar{t}$ production and so can be disentangled by fitting a single differential measurement. The dependence of the cross-section in each bin $j$ on the Wilson coefficients is given by:

$$
\sigma^{j}\left(C_{t G}, C_{t q}^{(8)}\right)=p_{0}^{j}+p_{1}^{j} \cdot C_{t G}+p_{2}^{j} \cdot C_{t q}^{(8)},
$$

where the parameters $p_{1}$ and $p_{2}$ are extracted from fits to dedicated EFT samples with terms linear in $1 / \Lambda^{2}$. Table 1 shows the expected and observed intervals for the nominal and individual fits compared to values from a recent global fit [8]. The stringent limits achieved on $C_{t q}^{(8)}$ demonstrate 
the data will be highly relevant in future global fits. The fitted central values are consistent with zero $\left(C_{t G}=-0.24, C_{t q}^{(8)}=0.03\right)$ [1], indicating there is no evidence of new physics in the data.

\begin{tabular}{c|c|c|c|c|c} 
Wilson coefficient & \multicolumn{2}{|c|}{ Marginalised 95\% Intervals } & \multicolumn{3}{|c}{ Individual 95\% Intervals } \\
& Expected & Observed & Expected & Observed & Global fit [8] \\
\hline$C_{t G}$ & {$[-0.44,0.44]$} & {$[-0.68,0.21]$} & {$[-0.41,0.42]$} & {$[-0.63,0.20]$} & {$[0.007,0.111]$} \\
$C_{t q}^{(8)}$ & {$[-0.35,0.35]$} & {$[-0.30,0.36]$} & {$[-0.35,0.36]$} & {$[-0.34,0.27]$} & {$[-0.40,0.61]$}
\end{tabular}

Table 1: Expected and observed 95\% intervals on the Wilson coefficients. For the marginalised results both Wilson coefficients are allowed to vary, while the individual intervals are extracted with the other coefficient fixed to zero [1]. The results are compared to the individual limits obtained in Ref [8].

\section{References}

[1] ATLAS Collaboration, ATLAS-CONF-2021-031, http://cds.cern.ch/record/2777237.

[2] M. Czakon et al., Top-pair production at the LHC through NNLO QCD and NLO EW, JHEP 10 (2017) 186, arXiv: 1705.04105 [hep-ph].

[3] ATLAS Collaboration, The ATLAS Experiment at the CERN Large Hadron Collider, JINST 3 (2008) S08003.

[4] M. Cacciari, G. P. Salam, and G. Soyez, The Anti-k(t) jet clustering algorithm, JHEP 04 (2008) 063, arXiv: 0802.1189 [hep-ph].

[5] G. D'Agostini, A multidimensional unfolding method based on Bayes' theorem, Nucl. Instrum. Meth. A 362 (1995) 487, ISSN: 0168-9002.

[6] M. Czakon and A. Mitov, Top++: A program for the calculation of the top-pair cross-section at hadron colliders, Comput. Phys. Commun. 185 (2014) 2930, arXiv: 1112 . 5675 [hep-ph].

[7] B. Grzadkowski, M. Iskrzynski, M. Misiak, and J. Rosiek, Dimension-Six Terms in the Standard Model Lagrangian, JHEP 10 (2010) 085, arXiv: 1008. 4884 [hep-ph].

[8] J. J. Ethier et al., Combined SMEFT interpretation of Higgs, diboson, and top quark data from the LHC, (2021), arXiv: 2105.00006 [hep-ph]. 